\title{
Clinical Trial Risk in Non-Hodgkin's Lymphoma: Endpoint and Target Selection
}

\author{
Jayson L. Parker ${ }^{1}$, Zoe Yi Zhang ${ }^{1}$, Rena Buckstein ${ }^{2}$ \\ 1. Biology Department, Master of Biotechnology Program, University of Toronto, Toronto, ON. \\ 2. Haematology/Oncology, Odette Cancer Center, Sunnybrook Hospital, Toronto, ON.
}

Received, May 11, 2011; Revised, May 29, 2011; Accepted, June 13, 2011; Published, June 13, 2011

\begin{abstract}
PURPOSE. To quantify the clinical trial risk of new drug development in Non-Hodgkin's lymphoma (NHL). Risk estimates for this disease have not been reported before. METHODS. We undertook a retrospective observational study of clinical trials in NHL in four subtypes to compare the success rate with the industry average. Our inclusion criteria required that a drug must have initiated its Phase 1 trial in one of the four NHL subtypes between 1998 and June 2008 in the US. In addition, clinical trials of new drug candidates that pertain to four subtypes of NHL were retrieved from clinicaltrial.gov. Drug candidates that did not meet these criteria were excluded from the study. RESULTS. The overall success rate $(8-11 \%)$ was significantly lower than the industry standard (17\%). Overall survival (OS) as a secondary outcome appeared more predictive than primary endpoints that were surrogate of overall success. Further, targeted therapies appear more successful in these lymphoma sub-types than broad acting drugs. CONCLUSION. Clinical trial risk in NHL, with an $89 \%$ failure rate reported here, may be reduced by basing decisions on OS secondary endpoints and the use of targeted therapies.
\end{abstract}

This article is open to POST-PUBLICATION REVIEW. Registered readers (see "For Readers") may comment by clicking on ABSTRACT on the issue's contents page.

\section{INTRODUCTION}

The average number of cancer therapeutic candidates entering clinical studies per year has more than doubled compared to 1990 (1) Despite such efforts, the US Food and Drug Administration (FDA) documents a steady decline in new drug approvals over the past ten years (2) and is at a 25 year low (3). Current attempts to estimate the risk of drug development have examined oncology outcomes from a selected group of firms, with estimates ranging from $6 \%$ to $8 \%$ of trial candidates eventually receiving FDA approval $(1,2)$. The current estimated risk adjusted costs to bring a new drug to market are of the order of US $\$ 800$ million $(4,5)$. Given these challenges $(6)$, it may benefit future drug development efforts in Non-Hodgkin's lymphoma (NHL) to know which clinical design decisions may increase the risk of drug failure.

While earlier studies examined the success rates of clinical trials in all disease areas (7) and all of oncology $(1,8,9)$ information about success rates in a specific disease area is not available. We limited our focus to four subtypes of NHL, of which treatment has improved significantly over the past
10 years: diffuse large B-cell lymphoma (DLBCL), follicular lymphoma (FL), mantle cell lymphoma (MCL) and cutaneous T-cell lymphoma (CTCL). We report the results of a review of drug candidates in the four NHL subtypes that were in clinical trials from January 1998 to June 2008 in the United States. The US is a major focus of drug development with just over half of drug sales derived from this region and is considered the top market globally (10). For these reasons, we chose to focus our search on the US market. To arrive at an estimate of risk of clinical trial failure in NHL, we compared successful drugs against the failed drugs during each Phase of clinical testing. This was an observational study, we did not attempt to interpret the statistical results of each trial and arrive at a conclusion of effectiveness. Observations are based on whether the drug in question did or did not pass to the next phase of clinical trial testing.

Corresponding Author: Jayson L. Parker, PhD, MBA, University of Toronto Mississauga, 3359 Mississauga Rd N., Mississauga, ON Canada; E-mail: jayson.parker@utoronto.ca 
In particular, we were interested in identifying possible clinical trial design choices that appeared to increase the risk of failure using the same methodology we applied to Crohn's disease (11).

\section{METHODS}

\section{NHL Drug Study Eligibility}

In this study, we collected data of all drug development programs that pertained to DLBCL, FL, MCL and CTCL between the periods of January 1998 to June 2008. The study included both drugs in a new combination with other drugs, and new drugs to be used along or in combination with standard care. While a search of clinicaltrial.gov yields many lymphoma 'hits' (over 1700), these 'hits' represent single trials, many of which belong to the same drug. We parsed through these 'hits' and systematically excluded drugs that were any or all of the following: a) initiated their Phase 1 trial for this indication before 1998; b) were not conducted by private sector; c) did not look at survival relevant outcomes or d) did not involve any of the four subtypes of lymphoma described above. Often the same drug can be tested in a range of trials with slightly different populations or with different concomitant medications at a single stage of development (e.g. Phase 1). Accordingly when we refer to a "drug" we include all the multiple trials for this compound (e.g. three Phase 1 studies involving the same drug is considered a single drug). Finally, drugs were classified as line extension if they previously have been approved for other indications and entered clinical trials for any of the four NHL subtypes in our time frame.

\section{Online Search Tools and Key Words}

Websites such as www.clinicaltrial.gov and the National Cancer Institute (NCI) were the primary data sources for this study. However, additional online database tools were used such as: www.archive-it.org ; PR news wire (Factiva, accessed through University of Toronto Libraries); Business Wire (Proquest 5000, accessed through University of Toronto Libraries); www.findarticles.com. Search terms used: diffuse large B cell lymphoma + clinical trial; mantle cell lymphoma + clinical trial; cutaneous $\mathrm{T}$ cell lymphoma + clinical trial; follicular lymphoma + clinical trial; Non Hodgkin's lymphoma + clinical trial; Non Hodgkin's lymphoma + trial + news; Non
Hodgkin's lymphoma + combination; Non Hodgkin's lymphoma + trials + press release.

\section{Clinical Trial Outcome Classification}

In this study we used a simple transparent rule set to classify clinical trial outcome as used previously (11). We made the following assumptions: a Phase 1 (and 1/2) clinical trial was classified successful if the drug advanced to a Phase 2 for the same subtype of NHL (but excluded in this study if instead it was advanced for another indication). A Phase 2 trial was successful if the drug had been advanced to Phase 3 clinical testing. Phase 3 clinical testing was successful if the drug received the FDA approval and remained on the market. There are two types of failure associated with clinical trials; medical failure and commercial failures. If a drug failed to meet its primary endpoint in Phase 2 and Phase 3 trials or had significant safety issues in any Phases that precluded further testing, then this was classified as medical failure, if this precluded further clinical trial testing in our time interval. Commercial failures were those drugs that had no signs of medical failure in press releases and conference proceedings, yet had no further clinical trial testing, for a minimum of 2 years. Commercial failures could be the result of competing program priorities, lack of financing or a downward revision of revenue forecast for the drug candidate in question.

In NHL, the overall survival (OS) is the most reliable clinical endpoint (12). However, for primary endpoints surrogate clinical endpoints are often used since they are believed to correlate with OS and are easier to demonstrate in a shorter period of time. In NHL clinical trials surrogate clinical endpoints based on classifications by the FDA are: complete response (CR), objective response rate (ORR), disease-free survival (DFS), progressionfree survival (PFS) and time to progression (TTP; $13,14)$.

The clinical trial success rate was calculated by determining the percentage of successful trials out of the total number of trials in a particular phase in MCL, CTCL, FL and DLBCL. Cumulative rates refer to the probability of completing the current clinical trial and any preceding clinical trial phase successfully (i.e. the product of probabilities).

Phase 2 trials with endpoints that were not specified in published papers were not included in the analysis of surrogate versus clinical endpoint 
analysis, thus yielding a smaller sample set of the data described here.

\section{Drug and Company Classifications}

In general, biologics were defined in accordance with classification by the FDA as "biological products are generally derived from living material-human, animal, or microorganism-- are complex in structure, and thus are usually not fully characterized" (15). A new combination of drugs was defined as the replacement of an essential ingredient of a traditional combination treatment with an alternative approved agent, but if this agent had not yet received FDA approval it was considered a new drug. Firms were classified as 'biotechnology companies' if they were listed on the index classification for that group of companies (Nasdaq Biotechnology Index) when they started Phase 1 clinical trials. Companies that were not listed on that index but had a market capitalization of over USD \$1B were classified as 'pharmaceutical companies'. Targeted therapy as defined by the NCI (16) as a type of treatment that uses drugs or other substances to identify and attack specific cancer cells.

\section{RESULTS}

A search of new drugs in clinicaltrial.gov that had entered at least one Phase I trial in one of the four NHL subtypes between January 1998 to June 2008 revealed 81 drugs, including 4 line extensions and three new combination studies using approved agents. Fifty-seven drugs had completed Phase I study between 1998 and June 2008 and the rest were on-going. Despite the large number of drugs undergoing clinical testing, we worked with a subset of these compounds given our inclusion criteria based on our time interval and four NHL subtypes. As demonstrated in Figure 1, a trend towards an increase in the number of the early stage trials was found during the period between 1999 and 2006. Forty-one out of 57 Phase 1 and 1/2 clinical trials tested drugs on more than one lymphoma histological NHL subtype, some even tested on other types of cancer. As the research entered Phase 2, the disease areas under study tended to be more specific to individual NHL subtypes: 14 out of 28 completed Phase 2 trials included only one NHL subtype.

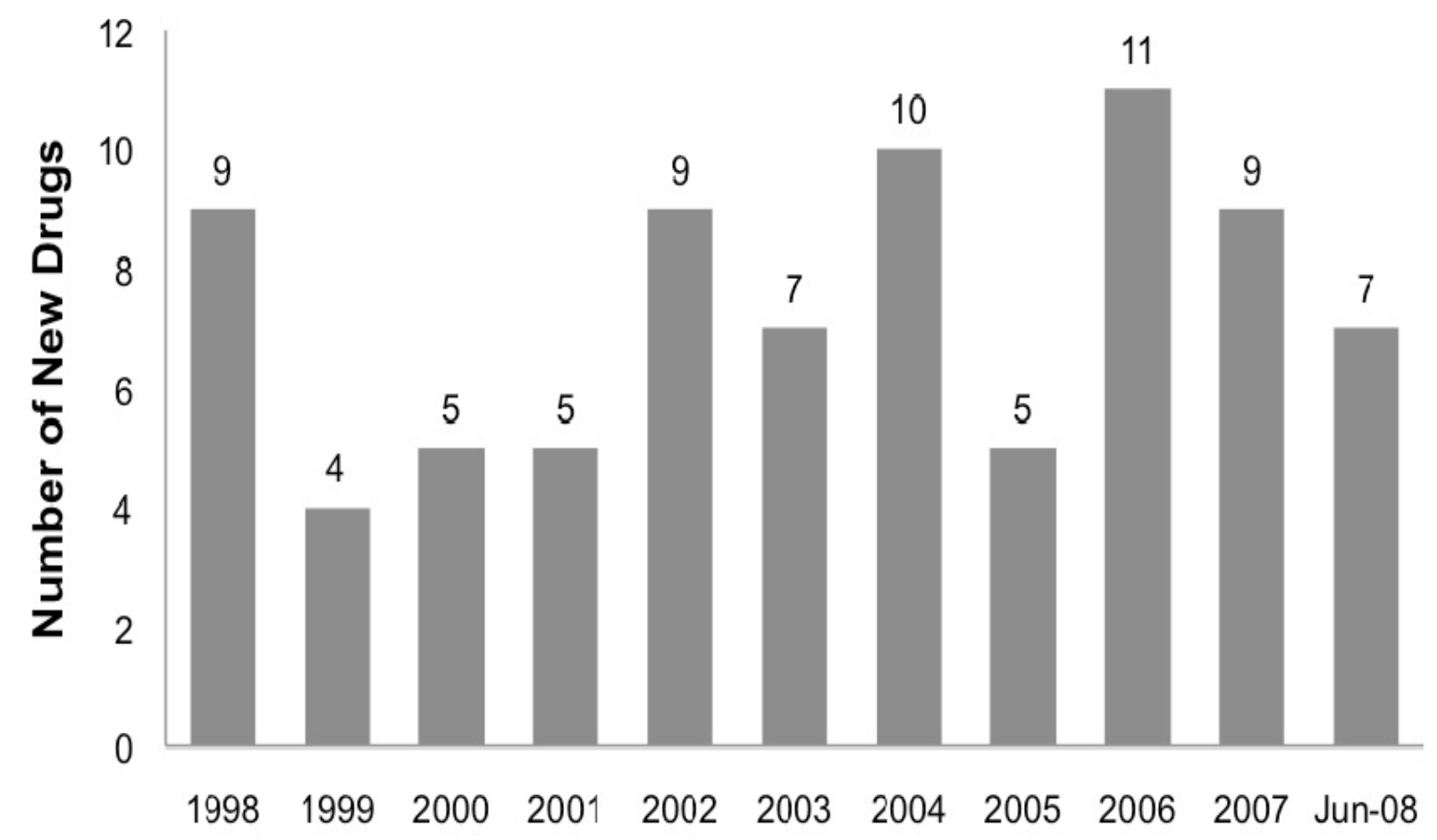

Figure 1. Depiction of drugs in the four types in NHL that met our screening criteria and their entry into clinical trial testing. Entry into clinical trial testing is based on the year of the first Phase 1 trial for the drug in question. 
The time interval examined in this study from 1998 to 2008 witnessed three new drug approvals by the FDA - vorinostat, rituximab (a new combination therapy and the only biologic approval), and bortezomib (17-21). Other approved drugs in this time interval were not included if their Phase 1 in one of the four NHL subtypes was before 1998. Vorinostat was approved by the FDA, after a Phase 2 trial, for the treatment of patients with CTCL. Although rituximab first received FDA approval in 1997 for the treatment of relapsed or refractory lowgrade or follicular B-cell (22), it was included in our research for its 2006 approval in new combination in CHOP therapy (cyclophosphamide, vincristine, prednisone, doxorubicin) or other anthracycline-based chemotherapy regimens for patients with previously untreated DLBCL $(17,18)$. Bortezomib, which was initially used for the treatment of multiple myeloma, had been granted full FDA approval as a line extension drug through a Phase 2 trial for the treatment of patients with MCL (21). Given our strict inclusion criteria requiring new combination therapies with approved drugs to have their corresponding Phase 1 trial in our time interval, very few qualified. New combinations included: FPD-R (pixantrone, fludarabine, dexamethasone, and rituximab); PCR
(Nipent, cyclophosphamide and rituximab); CPOP$\mathrm{R}$ (cyclophosphamide, pixantrone, vincristine, prednisone, rituximab). None of these combinations have so far entered into Phase 3 clinical trials.

Out of the 57 completed Phase 1 drug programs, 38 have successfully passed into Phase II. Fourteen of the 30 completed Phase 2 trials have entered Phase 3. Ten of these 14 trials were still ongoing in June 2008, while one of the four drugs that completed the Phase 3 trial has obtained FDA approval. In Figure 2, the Phase 1 and Phase 2 success rates of $67 \%$ and $47 \%$ respectively, were close to the industry standard of $64 \%$ and $25 \%$ (23). Therefore, the cumulative success rate for Phase 2 was $35 \%$ - similar to industry expectations of $31 \%$. Industry expectations were taken from previously reported rates for the industry as a whole (expressed as cumulative rates, taken from 1999-2004 data for a range of therapeutic areas (24).

The Phase 3 success rate was $25 \%$, yielding a low overall success rate of $8 \%$. Even when we include the two drugs that obtained approval after Phase 2 trials, the overall success rate in NHL is about $11 \%$, which is still substantially lower than the industry standard of $17 \%$.

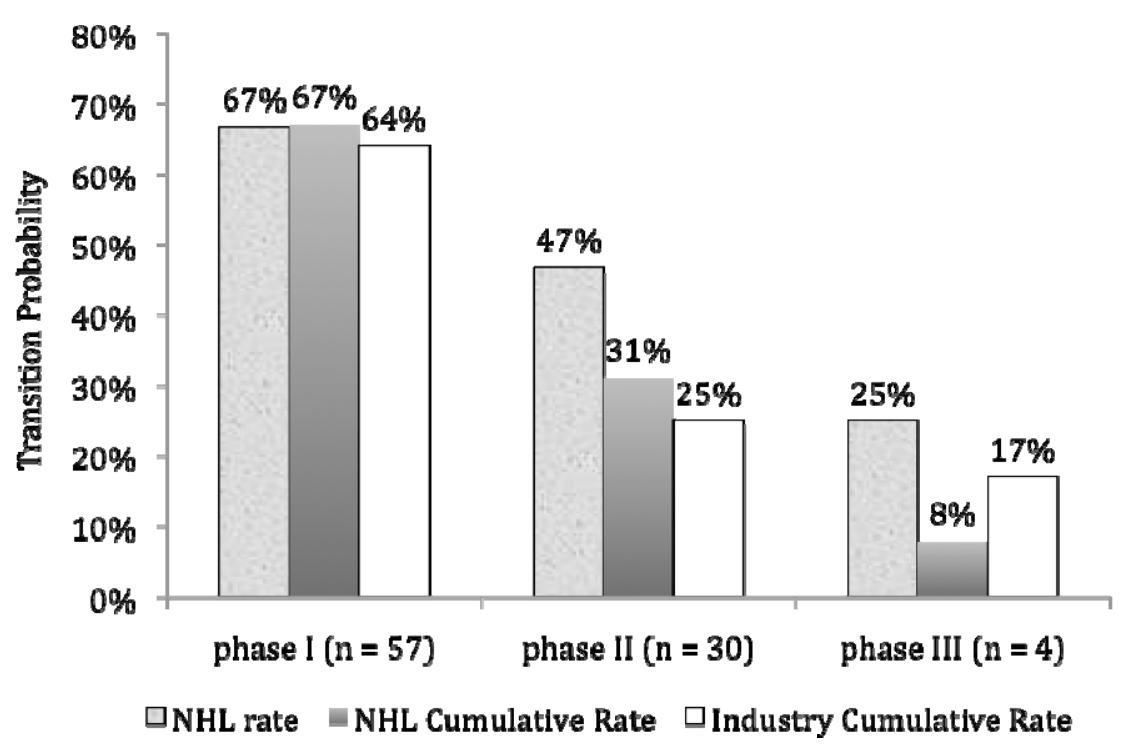

Figure 2. Clinical trial success rates in NHL. Drugs that entered Phase 1 clinical testing during or after 1998 were tracked up until June 2008. 'Pass rates' refer to the likelihood that drug would complete the current phase and advance to the next phase of clinical testing (or approval if currently in Phase 3). 'Cumulative pass rates' represents the product of probabilities for each prior phase, which for NHL is $11 \%$ for a product approval. Industry expectations are depicted as well from previously published studies (24). 
All the Phase 2 and Phase 3 clinical trials we analyzed had surrogate primary endpoints, and only a few included OS as a secondary endpoint (following the classifications of surrogate endpoints given by the FDA (2007)). Studies that reported event free survival, but not a specific breakdown for overall survival, were not included in this analysis. As shown in Figure 3,12 out of 30 Phase 2 trials $(57 \%)$ that used surrogate clinical endpoints successfully entered Phase 3 trials, compared with three successful trials out of the four $(75 \%)$ that included a secondary endpoint of OS. In Phase 3, all four trials used a surrogate endpoint as the primary indicator but only one succeeded $(25 \%)$. This successful trial included a secondary endpoint of OS (Figure 3). Overall, it appears based on this analysis, that surrogate endpoints appear to be less reliable indicators of success than the use of the OS endpoint. This difference existed despite the fact that OS was a secondary endpoint in the studies reviewed.

Clinical and commercial failure trends were depicted in each clinical phase in Figure 4. If we only consider medical failures as 'true' failure and add commercial failures to the list of clinically successful trials, then the success rates are $88 \%$ for Phase 1 (38 out of 43 trials were successful), 70\% for Phase 2 (14 out of 20 trials were successful), and $33 \%$ for Phase 3 (1 out of 3 trials was successful). The cumulative success rates then become $62 \%$ and $21 \%$ in Phase 2 and 3, respectively, which is substantially higher than cumulative success rates that factored in commercial failures (35\% and 11\%, respectively).

\section{Phase II Endpoints}

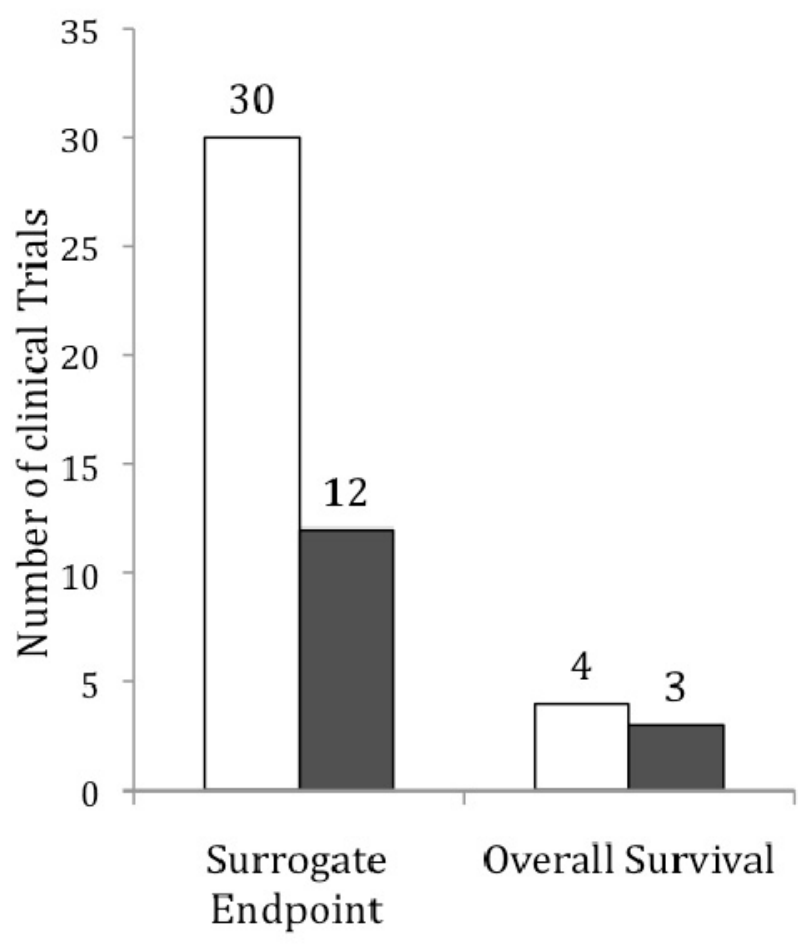

Total drug programs

\section{Phase III Endpoints}

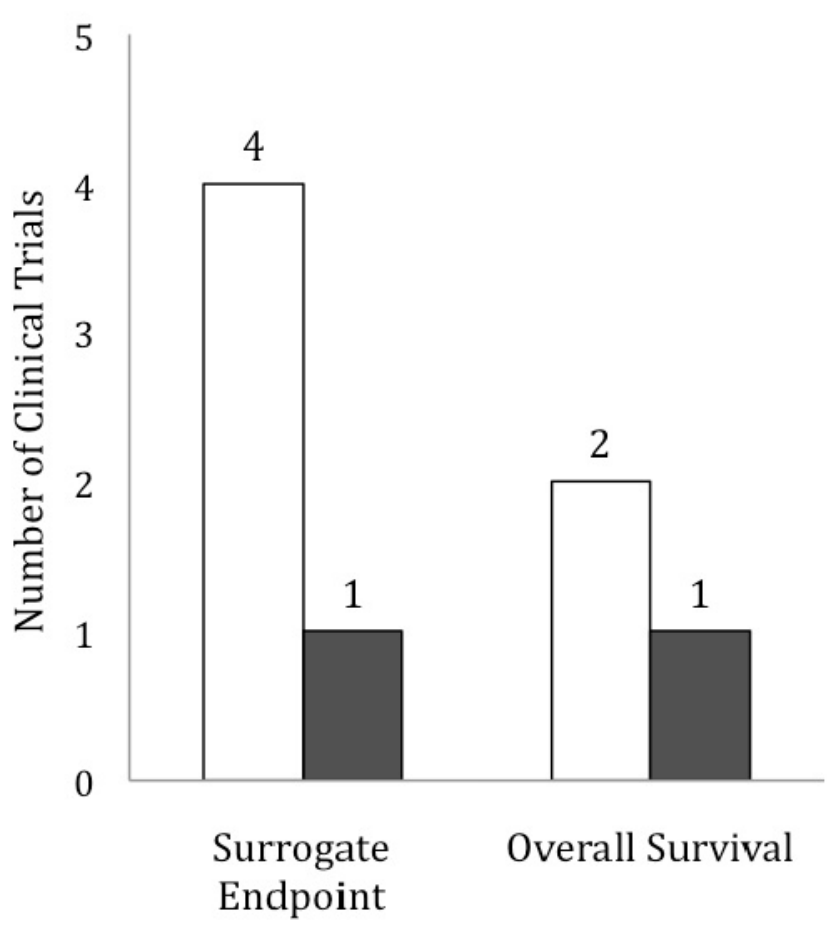

$\square$ Successful drug programs

Figure 3. Despite the small sample size, we attempted to analyze the impact of the choice of clinical trial endpoint on the success of a clinical trial in NHL. The FDA recognizes overall survival (OS) and a range of surrogate endpoints. This analysis contrasts the relative success rates between these two categories as predictors of the future success of compounds in NHL. OS is clearly a superior endpoint based on this dataset. 
Industry spent more effort to develop biologics than small molecules, exemplified by the 32 biologic drug candidates that entered Phase 1 clinical trials compared to only 25 small molecule drugs (Figure 5). In Phase 2 and Phase 3, biologic drugs had higher success rates than small molecule drugs. However, two small molecule drugs (vorinostat and bortezomib) were approved in our time interval compared to one biologic (rituxumab). Both small molecule drugs were approved based on phase 2 data (19-21). This suggests that in this indication, small molecule drugs have been more successful than biologics. We also classified the analyzed drugs into targeted immunotherapy and broad acting agents following NCI classification (15). Targeted therapy accounted for 58\% of all drug candidates in Phase 1 trials (Figure 6). Biotechnology companies introduced $91 \%$ of new drug candidates (based on ownership during Phase 1). Regardless of who sponsored the trials at later stages, drug candidates introduced by biotechnology companies showed a higher cumulative success rate $(11 \%)$ than their pharmaceutical counterparts $(0 \%)$ (figure not included).

\section{DISCUSSION}

Oncology is beset by problems facing the pharmaceutical industry, namely, difficulties with developing new agents to treat NHL. In this NHL study we quantified the risk of this process, and identified a few clinical design measures that may be predictive of success. The respective Phase 1, 2 and 3 success rates in NHL were found to be $67 \%$, $47 \%$ and $25 \%$, respectively (Figure 2). The overall success rate from Phase 1 to FDA approval was calculated as $11 \%$ in NHL, which is alarmingly low when comparing with the industry average success rate of $17 \%$ and more recent disease specific estimates for Crohn's disease at 19\% (11) and AIDs at $16 \%$ (unpublished data). The $11 \%$ success rate implies that only 1 out of 10 investigated drugs will successfully make it to market. However, the $11 \%$ rate reported here in NHL is still better than the $6 \%$ cumulative success rate which only considered large pharmaceutical companies (24), and the $8 \%$ cumulative success rate in all cancer therapeutic areas (1). Our search also showed that in the four subtypes of NHL, commercial factors contribute to clinical trial failures and suggests government funding could reduce this source of risk. Further, while OS, even as secondary outcome measures, appeared to be more predictive than primary surrogate endpoints.

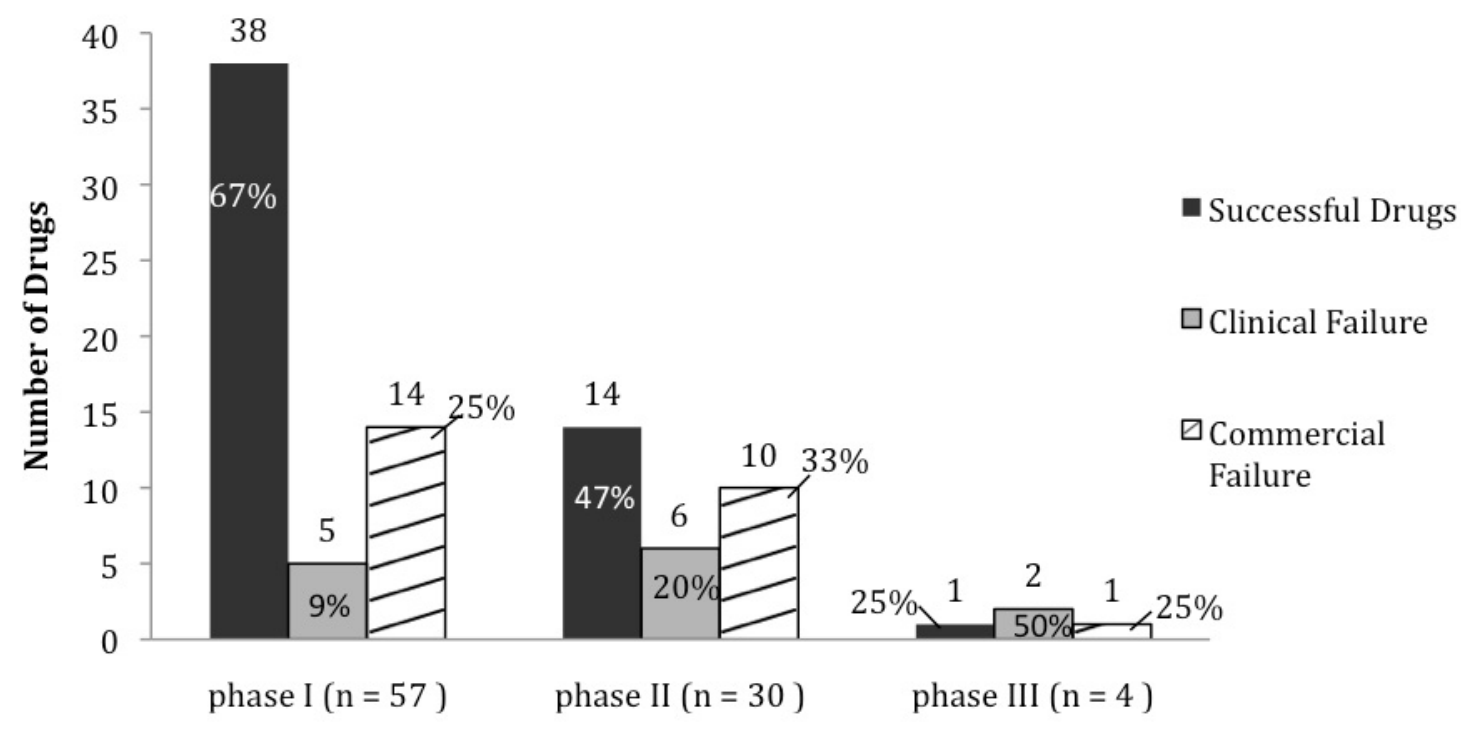

Figure 4. A classification of the types of clinical trial failure in NHL. There were a surprising number of commercial failures among drugs in early (Phase 1) clinical testing. This might suggest a role for government funding in this indication in early drug testing so that promising drug candidates are not lost. If a drug is not a failure or approved, it is ongoing in terms of clinical development as of March $1^{\text {st }} 2010$. 


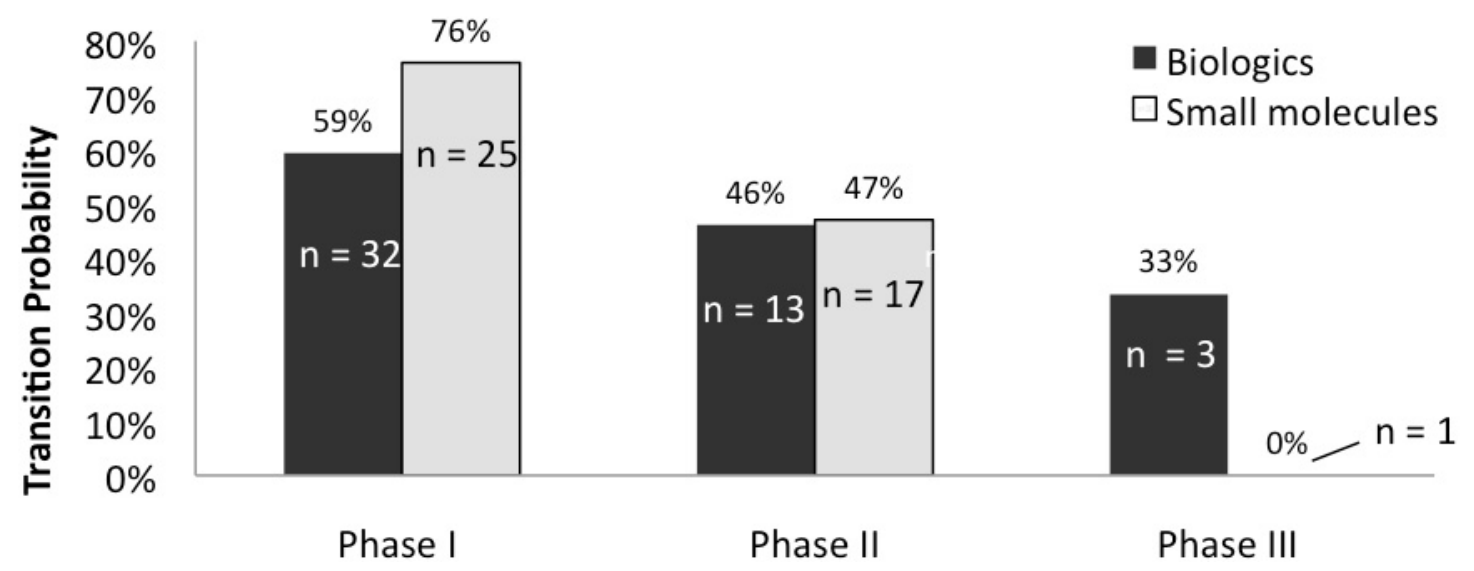

Figure 5. Clinical trial success rates in NHL based on whether the compound is a small molecule or a biologic, based on FDA definitions. There is a suggestion that biologics may be superior but it must be kept in mind there was a small molecule approved based on Phase II data.

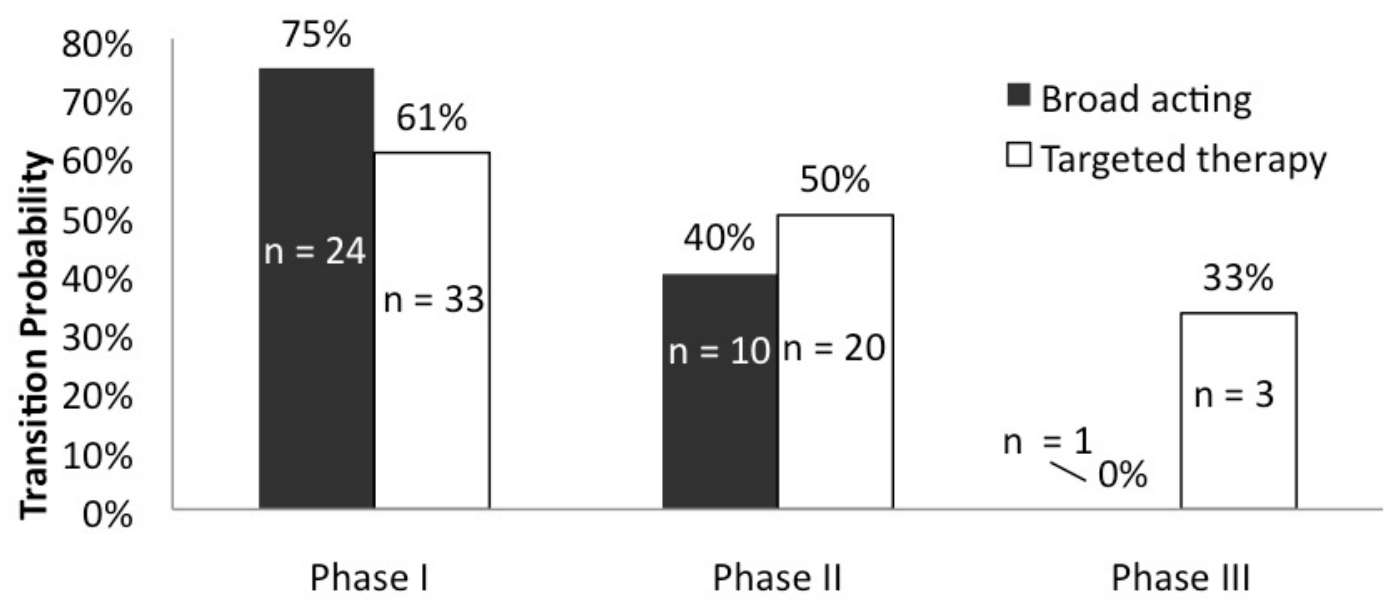

Figure 6. National Cancer Institute criteria for "targeted" versus "broad" acting therapies were used for this analysis. With this assumption, it appears there is a substantial benefit of targeted acting therapies over broad therapies in this indication.

Lastly, targeted therapies appeared to do well versus non-targeted ("broad acting") approaches (Figure 6) while small molecules appeared to outperform biologics. This last conclusion has to bear in mind that there were two small molecule drug approvals based on phase 2 data compared to one biologic approval based on phase 3 data.

Large drug development costs are incurred in clinical trials $(4,5)$. The estimated cost of a Phase 3 oncology trials is at least $\$ 10$ million over approximately 4.5 years $(25,26)$. While medical failure reasons are well understood, commercial failure reasons may include competing project priorities, sales forecast changes and other strategic changes for sponsor firms. As shown in our data, Phase 1 trials had a high commercial failure rate. As early as Phase 1 , commercial failures appear to play a strong role in NHL, which has not been observed in Crohn's disease (11). It's unclear why this is the case, it may be that NHL's historic difficulty as a disease area encourages manufacturers to cut their losses early at the sign of any trouble. When taking into consideration only clinical failures, by excluding commercial failures, we saw success rates in Phase 2 and Phase 3 were $62 \%$ and 21\% (as mentioned in the results for Figure 4). Commercial 
risks can be eliminated when a trial is sponsored by government funding, suggesting an important role in from public sector support in NHL drug development. However, this is a crude estimate, as some commercial failures may be, on closer inspection, a clinical failure as well.

The overall success rates from Phase 1 to FDA approval for biologics were lower than those of small molecule drugs (Figure 5). This is especially apparent in Phase 2 where we have taken into account the two small molecule drugs (vorinostat and bortezomib) that were approved based on Phase 2 data. The increased risk of biologics in NHL reported in this study may be indicative of a problem not with the technology per se but the choice of targets these agents are typically used to disrupt. Accordingly, the use of drugs that activate the immune system or interfere with growth factors needs to be optimized or better targets need to be found.

The endpoints we used to classify the Phase 2 and 3 trials were either surrogate endpoints or overall survival (OS). In our analysis of NHL trials, none of the Phase 2 and Phase 3 trials used OS as the primary endpoint and only four Phase 2 and two Phase 3 trials used OS for the secondary endpoint (Figure 3). However, the success rates of the trials with OS as the endpoint in Phase 2 and Phase 3 (75\% and 50\%, respectively) are much higher than those that used surrogate endpoints $(40 \%$ and $25 \%$, respectively). This trend suggest that clinical trial designs using only surrogate endpoints in NHL may not be worth the reduced cost and size of the trial and result in a higher risk of failure. This is a surprising finding that suggests despite the statistically underpowered role of OS as a secondary endpoint, this endpoint may be a more useful predictor of future trial success than statistically powered primary surrogate endpoints. Drug trials are usually statistically powered for one primary endpoint (27). In NHL a surrogate endpoint is selected as the primary endpoint and OS remains as a secondary outcome measure (12). This reflects the practical difficulties in recruiting enough patients to have a study large enough to have OS as a primary endpoint that is statistically powered (28). Therefore, decisions about late stage clinical testing based one the selected primary endpoint that is a surrogate marker, may be less reliable compared to secondary outcome measures such as OS. In other words, despite the fact such trials are not statistically powered for OS as an outcome measure, OS should be taken into stronger consideration in determining the success of the trial. This observation must be treated with caution, however, because it is not known whether the specific OS results were statistically significant. Ideally, this analysis would be performed based on whether OS was significant for the trial in question, but for now we can only refer to the broad association between trial designs that use OS as a secondary endpoint and whether the drug in question advanced to the next phase of clinical testing.

This retrospective observational study of success rates in NHL drug programs has many limitations. First, the sample size of the drug programs of NHL in clinicaltrial.gov is small. Second, we have not performed third party review of the data but have instead classified trials as successful if they advanced to the next phase of clinical testing. This simplifying assumption may warrant further study, but is well outside this initial report. Third, we weighted the surrogate endpoints response rate and EFS/PFS equally when in fact, they may have different predictive values for future success. Some of the differences we have identified are small and it remains to be seen, whether the trends identified here, will continue into the future in this indication with a larger sample size and a longer time interval of observation.

\section{CONCLUSION}

Our review of the reported clinical trials suggests that, in the four subtypes of NHL, real progress in the risk management of drug development can be achieved through designs that put more emphasis on the "efficacy signal" from secondary clinical endpoints like OS.

\section{CONFLICT OF INTEREST}

Parker consults with investment firms which may or may not have relationships with companies involved in this area, and has previously worked in the pharmaceutical industry. Buckstein receives industry funded clinical trial support for investigator sponsored studies and honoraria for advisory board and educational speaking engagements. 


\section{REFERENCES}

1. Rekhert JM, Wenger JB. Development trends for new cancer therapeutics and vaccines. Drug Discov Today 13:30-37, 2008.

2. US Food and Drug Administration. Challenge and opportunity on the critical path to new medical products.http://www.fda.gov/oc/initiatives/criticalpat h/ whitepaper.pdf, Retrieved on 2009-01-25, 2004.

3. Kaitin KI, DiMasi JA. Pharmaceutical innovation in the $21^{\text {st }}$ century: New drug approvals in the first decade 2000-2009. Clinical Pharmacology \& Therapeutics 89(2): 183-188. 2011.

4. DiMasi JA, Hansen RW, Grabowski HG. The price of innovation: New estimates of drug development costs. J. Health Econ 22:151-185, 2003.

5. Adams CP, Brantner W: New drug development: Estimating entry from human clinical trials. FTC Working Paper No. 262 262, 2003.

6. Honig $\mathrm{P}$ and Lalonde $\mathrm{R}$. The economics of drug development: a grim reality and role of clinical pharmacology. Clinical Pharmacology and Therapeutics 87 (3): 247-251. 2010.

7. Adams CP, Van Brantner V: Market watch : Estimating the cost of new drug development: Is it really \$802 million? Health Aff 25:420-428, 2006.

8. DiMasi JA, Grabowski HG, Vernon J: R\&D costs and returns by therapeutic category. Drug Inf J 38:211-223, 2004.

9. Munos B. Lessons from 60 years of pharmaceutical innovation. Nature Reviews Drug Discovery 8: 959968. 2009.

10. World Health Organization. The world medicines situation. 2004.

11. Parker JL and J. Clare Kohler. The success rate of new drug development in clinical trials: Crohn's disease. J Pharm Pharm Sci, 13(2): 191-197, 2010.

12. US Food and Drug Administration. Guidance for industry: clinical trial endpoints for the approval of cancer drugs and biologics. U.S. Department of Health and Human Services, Food and Drug Administration, Center for Drug Evaluation and Research (CDER), Center for Biologics Evaluation and Research (CBER), 2007.

13. Cheson BD, Horning SJ, Coiffier B, et al: Report of an international workshop to standardize response criteria for non-Hodgkin's lymphomas. J Clin Oncol 17:1244-1253, 1999.

14. Cheson BD, Pfistner B, Juweid ME, et al: Revised Response Criteria for Malignant Lymphoma. J Clin Oncol 25:579-586, 2007.

15. US Food and Drug Administration. Frequently asked questions about biological products. Accessed March $1^{\text {st } 2010 . h t t p: / / w w w . f d a . g o v / D r u g s / D e v e l o p m e n t A p p r ~}$ ovalProcess/HowDrugsareD...rovalApplications/The rapeuticBiologicApplications/ucm1 13522.htm
16. National Cancer Institute. Definition of targeted therapy - NCI Dictionary of Cancer Terms. http://www.cancer.gov/Templates/db_alpha.aspx?Cdr $\mathrm{ID}=270742$. Retrieved on 2009-01-25, 2009.

17. Feugier P, Van Hoof A, Sebban C, et al: Long-Term Results of the R-CHOP Study in the Treatment of Elderly Patients With Diffuse Large B-Cell Lymphoma: A Study by the Groupe d'Etude des Lymphomes de l'Adulte. J Clin Oncol 23:4117-4126, 2005.

18. Pfreundschuh M, Truper L, Oterborg A, et al. CHOPlike chemotherapy plus rituximab versus $\mathrm{CHOP}$-like chemotherapy alone in young patients with goodprognosis diffuse large-B-cell lymphoma: a randomised controlled trial by the MabThera International Trial (MInT) Group. Lancet Oncol 7:379-391, 2006.

19. Duvic M, Talpur R, Ni X, et al. Phase II trial of oral vorinostat (suberoylanilide hydroxamic acid, SAHA) for refractory cutaneous T-cell lymphoma (CTCL). Blood 109:31-39, 2007.

20. Olsen EA, Kim YH, Kuzel TM, et al. Phase IIB Multicenter Trial of Vorinostat in Patients With Persistent, Progressive, or Treatment Refractory Cutaneous T-Cell Lymphoma. J Clin Oncol 25:31093115, 2007.

21. Kane RC, Dagher R, Farrell A, et al. Bortezomib for the Treatment of Mantle Cell Lymphoma. Clin Cancer Res 13:5291-5294, 2007.

22. McLaughlin P, Grillo-Lopez AJ, Link BK, et al. Rituximab chimeric anti-CD20 monoclonal antibody therapy for relapsed indolent lymphoma: half of patients respond to a four-dose treatment program. J Clin Oncol 16:2825-2833, 1998.

23. J.A. DiMasi, L. Feldman and A. Wilson. Trends in risks associated with new drug development: success rates for investigational drugs. J Clin Pharm and Ther. 87(3):272-277, 2010.

24. DiMasi, L. Feldman and A. Wilson. Trends in risks associated with new drug development: success rates for investigational drugs. Clin Pharmacol Ther. 87(3):272-277, 2010.

25. Kola I, Landis J. Can the pharmaceutical industry reduce attrition rates? Nat Rev Drug Discov 3:711715, (2004)

26. Emanuel EJ, Schnipper LE, Kamin DY, et al: The Costs of Conducting Clinical Research. J Clin Oncol 21:4145-4150, 2003.

27. Roberts TG, Jr., Lynch TJ, Jr., Chabner BA. The Phase III Trial in the Era of Targeted Therapy: Unraveling the "Go or No Go" Decision. J Clin Oncol 21:3683-3695, 2003.

28. Stanley, K. Design of randomized controlled trials. Circulation 115(9): 1164-1169. 2007.

29. Hurvitz, S. A. Evolving options for the treatment of metastatic breast cancer: Progression-free survival as an endpoint. Cancer Treat Rev. Feb 9, 2011. 